\title{
A distinctive approach to obtain higher page rank through search engine optimization
}

\author{
M N A KHAN* and A MAHMOOD \\ Shaheed Zulfikar Ali Bhutto Institute of Science and Technology, Islamabad 44000, Pakistan \\ e-mail: dr.naeem@szabist-isb.edu.pk; dr.azhar@szabist-isb.edu.pk
}

MS received 12 August 2015; revised 24 January 2018; accepted 9 February 2018; published online 22 March 2018

\begin{abstract}
Search engine optimization (SEO) pertains to the activity of optimizing individual websites and webpages to get higher page rank in the search results. Websites are ordinarily optimized through back links, while individual webpages are optimized through specific keywords. In this paper, we propose a framework based on set of guidelines for keyword research analysis and back links generation. The proposed framework suggests that webpage content should be based on keywords and the site traffic should be monitored through referrals. We argue that if a website has some prevalent and pertinent keywords in its content and title along with a reasonable amount of back links that help watch the website traffic, then we can get better rank for the website in the search results. The framework also emphasizes that proper keyword selection and link building should be taken into account when developers and designers work on a software development project. The results of the study are reported here.
\end{abstract}

Keywords. Search engine optimization; electronic commerce; keyword analysis; back link watch; site crawl; web crawler; keyword density; site indexing.

\section{Introduction}

In the modern era, information technology has facilitated transmission of multi-facet information across the world. Internet community has emerged as a broad society comprising billions of users who use Internet as a communication tool on daily basis. Internet has transformed into a business network where a huge number of traditional business transactions are performed online. A large number of people, known as e-community, use Internet for marketing and business purposes [1]. There are millions of sites on the web and we heavily rely on the search engines for locating specific information and the targeted webpages. The discipline of Search engine optimization (SEO) started in the mid-1990s. Initially, SEO tactics were restricted to tagging the keywords and meta-keywords. The field of SEO started getting matured after 2003 when the strategies of anchoring text and interlinking pages of websites were introduced. Later in 2011, vertical search inclusion was introduced.

SEO is analogous to software that gets user queries and returns the required information to the user. SEO is used as a tool to attain higher search rank during online searches for the targeted websites. According to comScore survey [2], the biggest Internet community is in Turkey where $80 \%$ of the population use Internet for marketing and business. In

*For correspondence short, SEO mechanism is required to route the Internet search queries to the specialized websites having exclusive content. Hence, search engines play a critical role on steering users to the pertinent websites since $93 \%$ of Internet traffic is managed by search engines [3]. SEO is meant for those organizations and companies which want to stay competitive on the web. Millions of websites compete for placement in the search engines, therefore, only the websites improved and structured through SEO tactics receive increased visibility on the web.

SEO relates to the activity of optimizing individual webpages or the entire website to make it user friendly in order to get higher raking in the search results. Users generally visit those websites which appear on front page of the search results. Perhaps users may only examine the first 10 results shown on the front page and hardly few users move to the next page. With particular reference to SEO, it is important to fetch the targeted webpage at top of the front page in the search results. SEO is generally used to obtain higher page rank during compilation of the search results. SEO enables website to appear at top of the front page by using some pertinent keywords of interest. The optimized websites get better ranking in the search results and usually attract higher number of visitors. SEO process entails employing specific principles to improve search operation for websites and enhance probability of website discovery. To this end, Yang et al [4] stated that search engine marketing and business model has a strong positive relation 
with performance of the organizations. Yalcin and Kose [5] also asserted that SEO phenomenon has become a matter of significant importance for the corporate sector to promote businesses.

Search engine robots are based on various algorithms to link, rank and index websites. Efficient search engine allows users to accurately find the targeted information quickly. The major Internet search engines include Google, Yahoo, Ask, Yandex and Bing, etc. SEO technique consists of two processes: on-site optimization and off-site optimization. On-site optimization pertains to the practices that are used during the development of website. Off-site optimization relates to the practices through which the website and its contents are propagated over the Internet to increase its traffic which in turn enhance ranking of the website. SEO approaches that correspond to the guidelines followed by the search engines are known as "white hat" and the ones that violate the guidelines are termed as "black hat" [6].

Search engine bots crawl the web intermittently and perform indexing of the webpages based on certain keywords found in their content. Crawling the entire web is a long process due to extremely large volume of the web, hence, it is very likely that newly uploaded and updated webpages may not get indexed for months and do not appear in the search results. Although present day search engines use sophisticated algorithms, but there is still a caveat that these search robots cannot assess and appraise webpages the same way a human can perceive. To overcome this very limitation, SEO serves as a solution to figure out the nature of each webpage and determine how it can be made worthwhile to the users. SEO makes webpage easy for both search engine and users to cognize. SEO enables webmasters to affix appropriate clues and pointers with the website content so that a search engines can easily recognize what these contents are about. In this perspective, augmenting suitable structure to the webpage content is of paramount importance. Without optimizing the website content, it is very likely that a website remains invisible to search engines and may not reflect in the search results.

\subsection{Motivation}

Indexing the entire web is virtually not possible by the search engines. Even the webpages indexed by the search robots are not fully indexed; rather a very small part of the webpage content is indexed. Since indexing approaches varies from search engine to search engine, therefore, users occasionally use more than one search engine to find pertinent material of their interest on the web. Smart organizations want higher search ranking for their websites that always reflect the latest updated content; and this can only be achieved by optimizing the webpages using meta-keywords and applying backlink strategy, etc. Overcoming inherent difficulty of indexing webpages by the search engines served as a motivation to undertake this research. We have made an endeavor to help users to optimize their websites in a better way by using a three-pronged approach so that higher ranking for their websites can be assured. Addressing the three aspects of SEO process in a single framework is a novelty of our research work which has not been addressed in the earlier studies. Our study is a valuable endeavor to outline the three-pronged approach to facilitate users to significantly improve site optimization with lesser amount of efforts.

In this paper, we propose a meticulous SEO framework that encompasses different stages of the SEO process. The proposed framework aims at enhancing the current practices to obtain legitimate higher ranking for the websites. We argue that successful implementation of SEO not only requires on-page and off-page optimization, but also necessitates clean website structure so that it has no technical issue. The proposed framework fulfills the organizational needs for better management of SEO. Specifically, we emphasize that backlinks generation needs profound contemplation and regular revision to stay competitive. The experimental results show that our proposed framework yields promising results as more Internet traffic started getting routed to our sites. Our sites were properly indexed by the search engines and there were high number of visits for our sites and page views increased many-folds. In addition, average time spent by the visitors on our site increased significantly. Last but not the least, one of our site appeared at top of the first page of the search results and another acquired third position in the search engine results. These results were quite encouraging as studies show that first five results of the first page returned by the SERP account for $67.60 \%$ of all the organic clicks. We compared our results with the contemporary studies. Our results showed an overall $20 \%$ improvement as compared to the existing studies.

This paper consists of six sections. After an introduction to SEO in this section, we provide a brief description of the SEO process in the second section. The third section describes related work in the domain of SEO followed by outlining research gaps in this area. Our proposed SEO framework is presented in the fourth section. A case study based on experimental results are discussed in the fifth section. Finally, the conclusions are given in the last section.

\section{Search engine optimization process}

It is essential for web developers to build websites according to SEO standards. To grab attention of Internet users, SEO principles need to be scrupulously employed when developing a website because various factors are involved to move website upward in the search results 


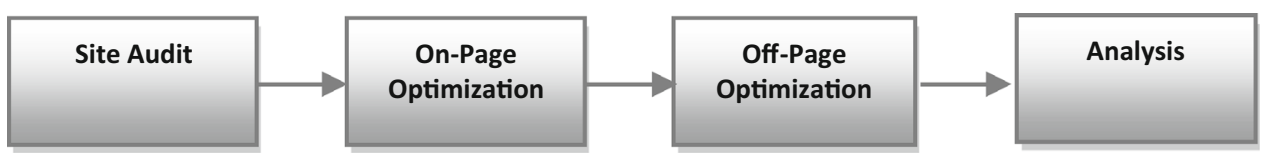

Figure 1. SEO lifecycle.

ranking. SEO mechanism works on backlinks and keywords which are valuable for website perspective. Search engine process entails the whole procedure of on-site and off-site optimization. The overall SEO lifecycle is shown in figure 1 .

\subsection{Importance of SEO}

Due to intensive use of Internet as a marketing channel, an electronic society has emerged which expects instant access to the relevant information. The Internet users expect to find relevant information on front page of the search results. SEO makes website prominent in the search results and higher ranked websites fetch more visitors. Higher ranking of a website provides an enormous opportunity to the online retailers to attract more customers. Hence, companies want a quick and ingenious mode for efficient marketing of their products, and this necessitates website optimization. A better position in the search engine rankings has a direct and positive impact on the frequency of users' visits to a website, thus bringing lucrative return on investment to the webpage owner.

\subsection{Keyword analysis and link popularity}

SEO is kicked off by analyzing keyword and link building. Keyword analysis necessitates that appropriate keywords pertinent to the website should be analyzed when optimization process is performed. Keyword analysis is based on the following procedure:

- Prepare list of potential keywords to be used for website optimization.

- Identify those keywords which can fetch a website in top 5 search results.

- Identify the external popular links to be included within the website.

There are some websites that provide keyword analysis and keyword density. Since a user generally uses two or three words to formulate a search query, therefore, the keywords density should be kept in the range of 5-7 words per 100 words used on a webpage. These words are normally used in meta-tags and page content. Link building requires using popular URLs for website optimization. Link building is typically performed after keywords analysis.

\subsection{Google, Alexa page rank and indexing}

Google Page Rank is an algorithm developed by Google to rank a website. Google keeps updating its spam fighting algorithms, such as "Penguin", "Panda" and "Hummingbird", from time to time to penalize those sites which do not follow its search optimization strategies. According to the webmaster tool, websites are ranked based on more than 200 factors. Though these factors have been kept confidential by the Google; but some of the factors such as site popularity, content quality, keyword density and publicity on social media have now become open secrets. Alexa.com also provides some useful information about a website. But, Alexa page rank has the following limitations:

- It only analyzes traffic that has Alexa toolbar installed.

- Websites are analyzed at their top level domain, which means that information about distinctive webpages is not analyzed at all.

Indexing is used for the swift retrieval of search results. Again, the websites are also indexed based on some keywords and phrases. This kind of information is captured from the meta-tags of a website.

\subsection{Key challenges}

Search engine optimization is a broader research area and a long lasting process. Acquiring and maintaining top position in search results is a challenging issue for SEO professionals. Relevant back-links generation is a time consuming and challenging activity. Considerable amount of time is spent on back-links generation which increases software project cost. Similarly, another challenging activity during on-site SEO is keywords selection since websites are optimized and indexed based on their keywords. Given higher volume of the web traffic, the selection of appropriate, relevant and less competitive keywords selection becomes a critical decision. The existing SEO tools do not provide enough support during any of these processes.

Search engines do not index webpages that are not publically available i.e., which are in SSL and HTTPS domains. A persistent issue for SEO is least awareness about website structures which results in lesser organic searches. Future changes to the search algorithms always remain mystery which is considered as a serious challenge because webpages currently having high visibility may 
move downward in the ranking. Also replicating the competitor's future activities how these would affect search activity in future pose a serious challenge. Another challenge pertains to the fact that application of SEO approaches and quality content in the webpages would not succeed if website has serious structural or technical issues. Duplicate content on several sites is another major technical challenge which necessitates cleaning up the sites.

The aforementioned challenges are framed from industrial and professional perspectives. From research perspective, we think that identification of SEO secrets such as models and frameworks, best practices and exploring specialized SEO tools and techniques are few additional key challenges.

\subsection{Tools for SEO}

Many commercial, open source and online tools are used to facilitate the SEO process. These tools are used for analysis of websites. This analysis provides insights and analytics about the website which helps developing potential SEO strategies. There are several tools that can be used for SEO. Some of them are:

- SeoQuake (an analytic SEO-tool that provides website information for a wide range of parameters such as page rank, Google index, Alexa and keyword density)

- Google Webmaster (shows visibility of webpages)

- Google Adwords (an online advertising tool)

- Google Analytics (generates statistics about website traffic and traffic sources, etc.)

- Google Trends (a powerful Internet marketing tool that allows users to see how often terms are searched on Google)

- Firebug (analyzes a website and provides capability for live monitoring of CSS, HTML and JavaScript on a webpage)

- Xenu (checks websites for broken links).

A number of marketing companies such as David Naylor, Search Marketing Gurus and Strategic Ranking provide services for website optimization. In addition, some of the common terms used in SEO are noindex, doindex, nofollow, dofollow and meta robots, etc. These terms refer to set of concepts and guidelines to optimize the websites. Site audits such as Moz Analytics and Ahrefs, etc. are commonly consulted by the software engineers to improve organic traffic, keyword rankings and visibility.

\section{Related work}

In this modern e-commerce era, everyone wants to crawl through the web and parse large amount of complex and diversified web content to find the most relevant results.
The online companies always seek higher visibility for their websites to attract more and more customers in order to promote their businesses. To enhance search visibility and ranking, webpages need to be optimized in an explicit way because search engine algorithms have distinctive policies to index information available on the web. Internal coding method and website content optimization are particularly important for retail and e-commerce sites [7]. SEO requires employing specific search engine friendly methods, tools and techniques. Some of the methods include optimization of images, optimization of web forms, structure optimization of frames, directory structures, URLs and optimization of flash media content. It is important to mention that these approaches generally fall in the category of friendly optimization methods and do not have any negative implications. Wang et al [8] employed feedforward backpropagation neural networks technique for search engine optimization to retrieve web information promptly. However, such an approach necessitates creating user profiles about their search patterns.

SEO also pertains to the retrieval of relevant webpages related to the pertinent user requests and in this connection, necessary filtering can help decrease the number of irrelevant pages in the search results [9]. Li-Hsing et al [10] reported certain improvements in the page ranking of a motel's website after implementing SEO techniques. Similarly, organizations like banks and government institutes can improve their web services by choosing suitable set of keywords [11]. Enterprises can use search engine for marketing because search engine optimization techniques enhance efficient retrieval of webpages [12].

Corporate sector and companies generally seek higher ranks for their websites not only to get hold of more and more business opportunities, but also to acquire worldwide recognition and popularity. In this regard, Elgharabawy and Ayu [13] investigated relationship between web content accessibility and webometric rank for acquiring higher page ranking. SEO techniques make websites prevalent in the search results and reverse engineering can be of much use for SEO analysis [14]. In semantic web, the web caching can help sort out the issue of website optimization at the architecture level [15].

SEO methodologies are administered in four key areas: content, keywords, structure and link optimization [16]. In the modern Internet world, searching for the targeted information necessitates that the website contents are aptly optimized by employing efficient SEO techniques. For this purpose, using the indexed webpages could be quite beneficial [17].

However, the tricky method of spamming the search engine is unscrupulously used to enhance website rank. In this regard, Somani and Suman [18] designed a black hat technique to counter SEO spamming. Google has also developed a mechanism to blacklist the sites that spam the search engine. The less thoughtful and aggressive application of SEO methods on a website could result in 
spamming. The increased popularity of sponsored search advertising could have serious implications for the Internet retailers e.g., Google searches may treat such websites as spam and filter them from the search results.

Egele et al [19] developed a classification technique that extracts important features and keywords from websites and then based on the extracted features it distinguishes spam websites from legitimate entries. The proposed technique is quite useful to remove spam websites from the search results and only those webpages are shown to the users which contain useful content. In this regard, Ayanso and Karimi [20] stated that keyword competition could only have moderating effects on the web searches and only those keywords should be included in the webpages which are based on consumer search behavior. Furthermore, black hat websites are likely to be banned or penalized by the search robots, but many SEO tools employ black hat approaches and sometime trickily deceive the search engine by imitating to be white hat approaches [6].

To achieve better performance, Ortiz-Cordova et al [21] suggested for using internal queries on top of the external query results acquired through a search engine. "Metasearch engine" which combines results of multiple search engines provides a considerable improvement both in website coverage and search effectiveness. Amin and Emrouznejad [22] proposed a linear programming mathematical model for optimizing the ranked list results obtained through multiple search engines. Keeping in view the impact that search engines inflict on the search results, businesses and corporate sector across the world tend to optimize their websites to compete with their rivals [3]. In addition to user-centric focus, the modern search engines now integrate semantic web primitives to enhance better insight into the web content. The objective of SEO mechanism is to enrich and alter the content of a website to achieve its optimal ranking in search engine results [23]. Obtaining top ranking in search engine result pages has become an acute requisite of the business sector. Pan [24] states that majority of the online tours and travelling agencies use search engines to seek most suitable travel itineraries that meet their clients' desires. In this regard, organic clicks play a vital role.

Organic and sponsored search results have always been a bone of contention among the search marketing advertisers. Berman and Katona [25] highlighted importance of organic and sponsored search results by stating that these strategies help improve search engine's ranking quality and argue for investing more on sponsored links. Since each search engine uses different approach for composition of search result pages, therefore, organic results are undermined by shortcuts and advertisements. Organic, sponsored, shortcuts, primary search results, prefetch, snippet and child are seven popular types of search engine results. Höchstötter and Lewandowski [26] investigated the composition of search engine results and reported that Yahoo and Google rank search results according to their own offerings such as Yahoo Answers and YouTube.

SEO techniques have also been tried to optimize academic websites and scholarly literature such as Google Scholar. To this end, Beel et al [27] discussed detailed guidelines for optimizing websites pertaining to scholarly literature. SEO strategies have also been applied in the domain of online newspapers, online news channels and news websites to gain high visibility in search engines results. Such an approach helps journalists to search the relevant information in an effective manner and promotes Internet journalism [28]. Understanding features of search engine algorithms is necessary so that pertinent tactics and counter strategies can be devised and duly implemented at the time of website development in order to fetch higher search rankings for the websites [12].

\subsection{Research gaps}

SEO practitioners and Google emphasize that search engine rankings can be enhanced by improving quality of the site content. A significant research gap is that existing literature does not address the importance of site content in the SEO process. Another research gap is that the existing academic literature is not in line with the white papers published by Google and other SEO companies. Further, the intersection of site content and specific keywords used on it is not addressed in the existing literature. Identifying the relevance of the keyword with respect to the site content is also a key challenge in this area. Another caveat in SEO practices published in the academic articles is that that these practices mainly focus on the marketing perspective. However, we think that SEO tactics can be improved by deliberating on psychological perspectives of SEO. In view of this, we propose a novel SEO framework in the subsequent section that addresses aforesaid challenges.

\section{Proposed SEO framework}

Our proposed SEO framework is shown in figure 2. Our proposed SEO process starts from site audit followed by analysis based on on-page and off-page optimization. At each phase of our proposed framework, we outline pertinent guidelines to be undertaken to optimize the sites. In this section, we outline specific steps and guidelines for each of these phases starting from the site audit.

\subsection{Site audit}

The first step is to conduct site audit before starting SEO activities for a website. In this phase, a site is thoroughly analyzed against specific parameters and based on this 


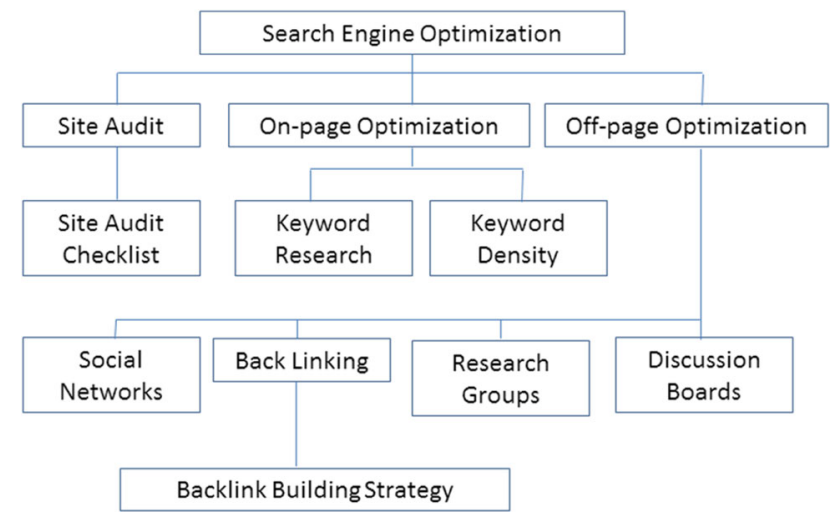

Figure 2. Proposed SEO framework.

analysis the specific changes are suggested. Table 1 presents our proposed SEO site audit checklist.

The detailed description of the site audit components is provided in the subsequent paragraphs. We suggest to implement these components as per their recommended use in the following paragraphs.

(a) Total Readable Content: Website contents are considered as a key substance in SEO. Web surfers do not take interest in the site if there are no interesting, original and relevant content on the webpage. A number of online tools are available that can provide statistics about readability of the site content.

(b) Internal Links: Internal links are hyperlinks that refer to other pages on the same domain. Internal links can be built strategically because they tell search engines about the preferences and thematic dependency of the webpages. So, we need to think logically before creating internal links.

(c) Average Speed: Average speed refers to the page load time and it is an important factor that search engines, especially Google, consider while ranking a site. If a site contains large amount of data, then its page load

Table 1. SEO site audit checklist.

\begin{tabular}{lc}
\hline Sr \# & Site Audit Checklist \\
\hline 1 & Total Readable Content \\
2 & Internal Links \\
3 & Average Speed \\
4 & Broken Links \\
5 & Canonical URL Check \\
6 & Flash, Frames and Ajax \\
7 & Google banned URLs \\
8 & Site Map \\
9 & Site Index \\
10 & Tags and Meta Tags \\
11 & Competitor Analysis \\
12 & Search Engine Page Results \\
\hline
\end{tabular}

time will increase. Moreover, large amount of JavaScript, CSS files and images also increase the page load time.

(d) Broken Links: Broken links are internal or external website links that do not exist on the web any longer. Presence of these links has a bad impact on the site ranking. Many broken links checker tools such as Xenu are freely available on the Internet.

(e) Canonical URL Check: Canonical URL means multiple URLs referring to the same page. Canonical pages have almost similar content. The best practice from SEO perspective is to specify canonical pages with canonical URL tag.

(f) Checking Ajax/Frames/Flash Use: The websites which use a lot of Frames, Ajax, Flash and other multimedia content never become search engine friendly as they cannot be crawled and indexed easily. Such sites have lower visibility and low page rank.

(g) Checking Google Banned URLs - Penalization Verification: In case the Google Webmaster guidelines are violated during the construction of a website, then Google may ban that site or some of its pages. If a site has banned pages or backlinks then it may affect the site ranking and visibility. Banned pages and backlinks may be checked with banned URL checker tools.

(h) Sitemap: Sitemaps are an easy way for webmasters to inform search engines about specific pages on their sites that are available for crawling. A sitemap is an XML file that lists URLs for a site along with additional metadata about each URL so that search engines can intelligently crawl the site. The metadata include information such as when it was last updated, how often it usually changes, and how relatively it is important to the other URLs within the site.

(i) Index Ratio of Site: Google has a secondary index containing those pages which are considered to be less important. These pages are considered supplementary and are returned in SERPs (Search Engine Results Pages) only if no pages are found from the main index matching the search terms. The Supplemental Index Ratio tells what percentage of pages indexed from the website are supplemental. The lower Supplemental Index Ratio is better. The supplemental index is a secondary index for lower ranking pages. Pages found in the supplemental index tend to be crawled less often and are never assigned a page rank. As a result, these pages tend to appear at the bottom of the organic search results.

(j) Tags and Meta-Tags (Title, Page Description, Keywords Heading, Robots): Meta-tags provide information about a site. HTML heading tags and meta-tags should contain the targeted keywords. Webmaster recommends using $\mathrm{H} 1$ for heading-tags. Meta tags are included inside the $<$ header $>$ tag. It is recommended to add meta-title, meta-keywords and meta-description 


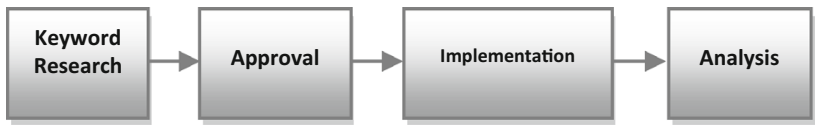

Figure 3. On-site optimization.

tags. Meta-robots tag may also be used if needed. The purpose of this tag is to ensure whether the web page is to be crawled/indexed or not. Use of this tag is optional and should be used if content have been disallowed in Robots.txt file.

(k) SERP Search Engine Results Page: Millions of results can appear as a hit when we supply a search query to the search engine, and this is called SERP. There are two types of SERP listing: organic and paid. Organic are the natural listing while the other ones are the paid advertisements.

(1) Competitors Analysis: SEO Competitors Analysis is the study of identifying competitors for the same keywords used in a website. Through this analysis, one can know how its competitors have optimized their website, what are their targeted keywords, how many backlinks they have, and where their backlinks are stored, etc. Competitor analysis helps us to optimize our website in a better way.

\subsection{On-site optimization}

In this phase, we propose our approach to optimize the website internally based on the site audit. The main activity in this phase is keyword research. A list of suggested keywords needs to be prepared by the analyst based on the suggestions/feedback sought from the client. After this, website should be internally populated with the keywords by implementing them on-site everywhere i.e., within titles, tags, metatags and content of the webpages. After implementing the keywords, website should be analyzed on regular basis to check the expected results. During this analysis, three main artifacts should be closely observed which include site traffic, page rank and SERP. Google analytics are normally used for traffic analysis. Based on this analysis new strategies can be developed for further improvements of the site to make it more search engine friendly. On-site optimization process is depicted in figure 3.

We suggest the following guidelines for on-site optimization:

- Domain Name and Title of the website/webpages should contain pertinent keywords for which the website is required to be optimized.

- Write a short description of the website. It should contain the appropriate keywords/indexed terms.

- Figure out keywords through which website is meant to be optimized and put these keywords in headings, anchor tag, Alternative (Alt) tags and in the main contents. Keywords are selected after a thorough analysis. Many aspects like competition, local and global traffic and trends are analyzed while selecting a keyword. Google AdWords and Google Trends are the commonly used tools for keyword research.

- Create a perfect architecture of the website that can be easily crawled. Use latest tools and software for website development. Poor website structure undermines all the SEO activities.

- Regularly update website with fresh and unique content so that the websites content should comply with the ever changing Internet trends. Regular website updating is necessary to counter strategies used by the competitors from time to time.

- Ensure that webpages contain the latest keywords/ buzzwords that are more relevant to the website content.

- Video, images, audio, infographics and other multimedia content should also be optimized.

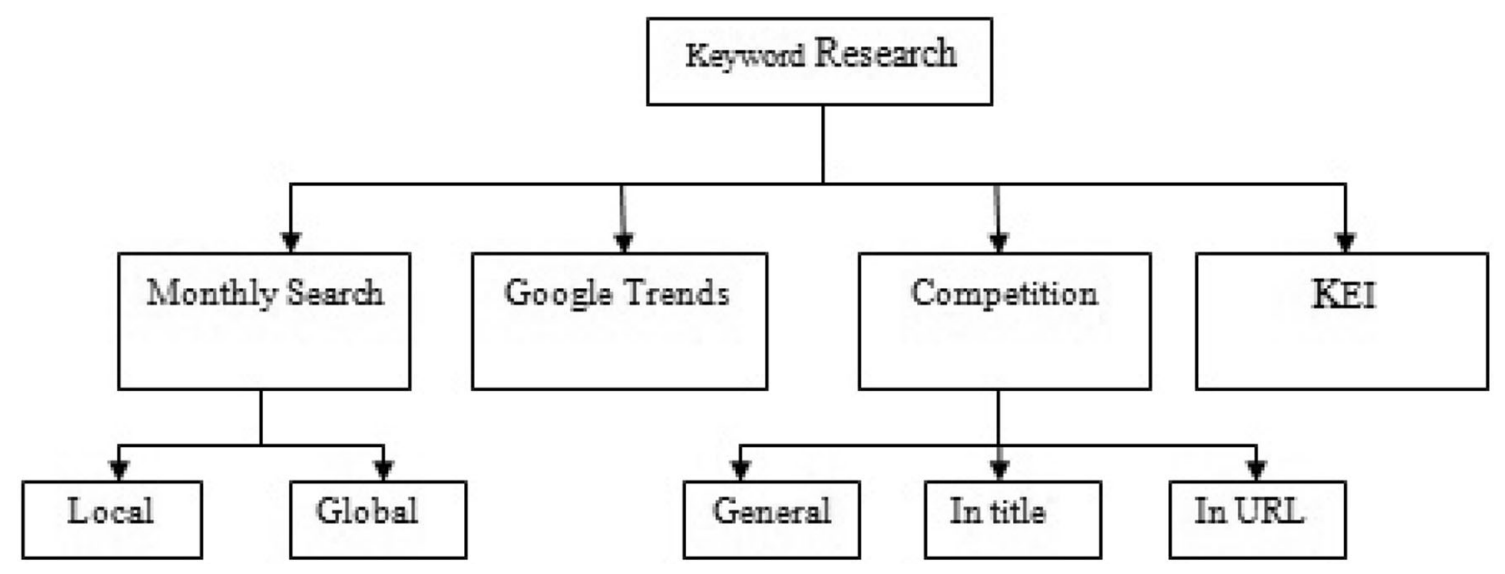

Figure 4. Keyword research. 


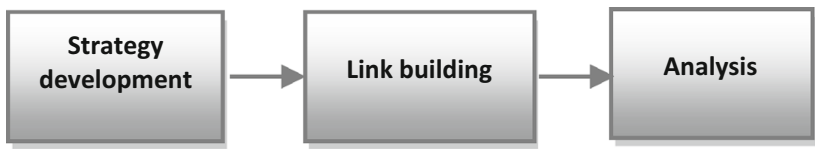

Figure 5. Off-site optimization strategy.

- Use keywords in the title and header tags of webpages.

- Webpages content should be populated with only those suitable keywords which do not violate the webmaster rules.

4.2a Keywords research: In keyword research, we find out monthly local and global searches, Google trends, SERP keywords general competition, in-title and in-URL competition. Appropriate keywords selection is a challenging task in SEO. Optimized webpages consist of specific keywords as appropriate keywords facilitate users to find the relevant-information and this procedure helps get higher ranking for webpages. In figure 4 , we present a framework for keywords analysis.

We also recommend using keywords effectiveness index (KEI) for selecting the effective keywords. There are several tools available for keyword research that provide an indepth analysis. The best keywords are those which have high probability of searches and low competition. KEI can be measured with the following equation.

$\frac{\text { MonthlyLocalSearches } \times 2 \times 1000}{\text { SERP }}$

4.2b Keyword density: Appropriate keywords are populated within the content of a webpage to make it search engine friendly. Ideal keywords density for the entire content is considered to be $5-8 \%$. Density of keywords makes the site content keyword-rich and relevancy for search engines is also increased.

\subsection{Off-site optimization}

Off-site optimization relates to the practices through which the website and its content are propagated and proliferated over the Internet to enhance Google ranking of the website and better positioning in SERP. Off-page optimization strategy should be developed based on the SERP and competitors analysis performed during the site audit. The main process in this phase is link building and sometimes this process is called backlinks generation. Figure 5 illustrates the off-site optimization strategy.

We suggest the following guidelines for off-site optimization:

a. Back Linking: Place website links on other well-reputed and top ranked websites. This helps enhance page rank. Some additional suggested course of actions include:

- Guest Article and Blog writing
- Forum Posting and Blog Commenting

- Directory Submission and RSS Feed

- Try to get ".gov" and ".edu" links

- Social bookmarking

- Ensure active existence on Social Media

b. Social Network: Place links of the social networking websites such as Twitter, Facebook, LinkedIn and Google + on your website. Proliferate website content on these social networking sites by creating your weblinks/webpages on these sites. This tactic helps other people to visit your social media profiles and ultimately visit your website.

c. Groups: Join groups such as Yahoo, MSN, Google, etc. and post comments on these groups. The posted comments should always have a reference/link of the targetted website or the specific webpage that we want Internet users to read. Sending promotional emails to Internet users is also a part of this strategy.

d. Join online community and discussion boards: place comments containing links of your website on these sites.

e. Use trusted websites to share your links and also insert links of the reputed sites in your own webpages: An important consideration during this process is to strictly adhere to the Google webmaster guidelines because Google is the major search engine and more than $80 \%$ of web searches are made on Google. Noncompliance to Google webmaster guidelines may result in either blocking or penalizing of the website by the Google.

\subsection{Backlinks generation}

Backlinks, also called the referral links, are the incoming links to a website from other webpages and this is an important fragment of the off-page optimization. Backlinks enhance traffic to a website and increase its visibility. Therefore, it is necessary to get some backlinks from wellknown social and community websites such as Twitter, Facebook and LinkedIn. In addition, backlinks from the sites in ".edu" and ".gov" domains also dole out good impact on website visibility. Always try to get backlinks from the sites that are more consistent and interconnected to your website. Figure 6 illustrates our proposed backlink building strategy.

The detailed description of elements of backlinks building strategy is provided below.

a. Forum Posting: pertains to participating in a discussion forum by placing comments on the threads. This is a good way to route Internet traffic to the targeted website. Two things should be kept in mind while commenting on discussion forums. Firstly, the comments should add something new into the thread as otherwise administrator 


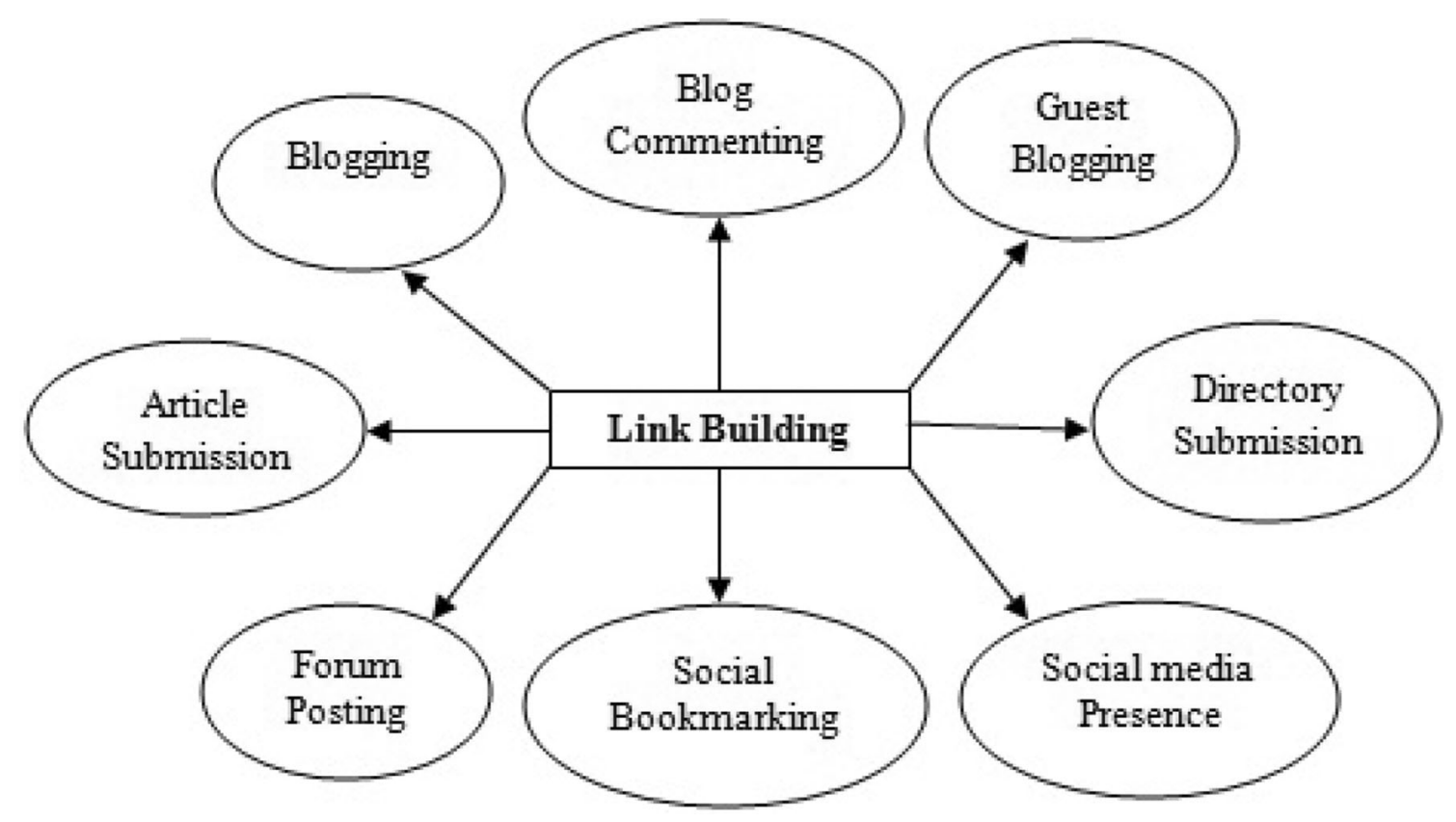

Figure 6. Backlinks building strategy.

will not approve the comments. Secondly, we should add a signature that refers to our website.

b. Blogging: means writing blogs to keep visitors informed and to stay in touch with them. It is suggested to provide unique, informative and quality content to the audience.

c. Guest Blogging: refers to writing and posting an article on the blog owned by someone else. This is an effective technique for diverting traffic to our website. For this purpose, we need to request a blogger to post our article on his/her blog through social engineering.

d. Blog Commenting: is another way to get traffic and make our website popular. For blog commenting, we need to work elegantly as often there are bunch of comments pending with the administrators. If our comments are not valuable, then administrator may not approve and publish the comments. To keep ourselves live on the discussion forums, we should always add something new in the blog discussions. If we like or dislike something then we should write some pertinent comments to justify our stance. We should always supply the desired links tactically without giving the impression of promoting our website. While commenting on blogs to gain search engine popularity, verify whether the blog belongs to dofollow or no-follow category. If we are commenting just for traffic generation then it does not matter whether the blog is do-follow or no-follow.

e. Article Submission: sites provide opportunities to submit articles and generate backlinks for our website.

f. Directory Submission: is the process of submitting site URL with targeted keywords and description to the web directory. This can help us make our website popular. g. Social Bookmarking: is also a valuable link building strategy. Social bookmarking sites provide an online place for bookmarking and tagging a website. These bookmarks are publically available. Search engines visit bookmarking sites more frequently, so it is an effective and fast way of making a website popular in search engines. Twitter (social networking site), Delicious (a tool to save, organize and discover interesting links on the web), StumbleUpon (a tool for sharing website and web contents with others users), Reddit (a social news and entertainment website), Digg (social news site) and Pinterest (a tool to create and manage theme-based image collections such as events, interests and hobbies) are few examples of social bookmarking sites that facilitate promoting our websites.

h. Social Media Presence: means placing links of our website on social media. This helps increase the number of visitors to our site.

\section{Case study: Framework validation}

We have implemented our proposed framework on seven SEO projects in a medium-sized organizations which provide SEO services. The team consisted of four members: two SEO engineers, one senior social media marketing expert and a team lead. The SEO team also coordinated with the development team and the designer team. There were four members in the development team while the software design team consisted of two designers. The SEO team worked simultaneously on all the seven projects. 
Before applying the strategy summarized in our proposed framework, the keywords were selected blindly. There was no on-page optimization, just few off-page optimization techniques were applied tactically. The purpose of applying our proposed framework was to follow a proper strategy to streamline every process in a systematic manner. We started from the scratch and analyzed all the websites with our proposed SEO audit template. When we audited the sites, there were many shortcomings from SEO point of view. Some of the major and common issues that we identified in the audit review are reported below.

There was very less traffic on the sites especially the organic traffic was incredibly negligible. Page rank was sometime zero and sometime one. SERP positioning was far away, our sites were not listed even in the first 100 pages. There were several keywords used in the title but none of them were being targeted properly in the content. Keywords density and site index ratio were terribly low. The main reason for low search engine visibility of our sites was that our sites were not using canonical URLs and there were no sitemaps on the sites. The "H1" tag of HTML was used less frequently and even wherever it had been used it was not optimized at all. In some cases, robot.txt file was found on the server but no robot tag was used on any of the page. Keywords used in the title and description of the webpages were very generic, therefore, theses webpages suffered from the competing websites. To overcome these issues, we used category, sub-category and thematic keywords representing domain and business ideas as discussed in our proposed framework.

After completing the audit, we worked on our on-page optimization strategy. We performed a thorough keyword research and populated our sites with pertinent and targeted keywords. After that we started developing and implementing an off-page optimization strategy. By following our framework, we performed a meticulous link building and ensured social media presence for our sites.

Meanwhile, we kept on analyzing the search engine results for our sites. We noticed that on-page optimization quickly started producing promising results in terms of improving the page rank. After one month when we had implemented our on-page optimization strategy, the page rank for our website increased to "4". This showed promising results for our proposed framework implementation.

Similarly, after implementing our off-page optimization strategy, we noticed that its results started delivering promising returns slightly later. The reason for this delay was due to the fact that some of the processes in off-page optimization took longer time like finding relevant link building sites, and similarly the blog commenting and directory submission also took longer to get approved and published. These restrictions are some of the major challenges that prevent off-page optimization to provide quick and efficient results. However, the enhanced results can still be achieved if our proposed framework is followed. After
Table 2. Experimental results.

\begin{tabular}{|c|c|c|}
\hline & $\begin{array}{c}\text { Traffic overview (before } \\
\text { framework } \\
\text { implementation) }\end{array}$ & $\begin{array}{c}\text { Traffic overview (after } \\
\text { framework } \\
\text { implementation) }\end{array}$ \\
\hline Referrals & 316 & 2,527 \\
\hline $\begin{array}{l}\text { Direct } \\
\text { Traffic }\end{array}$ & 75 & 3,436 \\
\hline \multirow[t]{2}{*}{$\begin{array}{l}\text { Search } \\
\text { Engines } \\
\end{array}$} & 0 & 16,953 \\
\hline & $\begin{array}{l}\text { Visitors overview } \\
\text { (before framework } \\
\text { implementation) }\end{array}$ & $\begin{array}{c}\text { Visitors overview (after } \\
\text { framework } \\
\text { implementation) }\end{array}$ \\
\hline Visitors & 391 & 22,916 \\
\hline $\begin{array}{l}\text { Absolute } \\
\text { Unique } \\
\text { Visitors }\end{array}$ & 281 & 18,228 \\
\hline $\begin{array}{l}\text { Returning } \\
\text { Visitors }\end{array}$ & 121 & 5,573 \\
\hline Page views & 763 & 111,541 \\
\hline $\begin{array}{l}\text { Average } \\
\text { Page } \\
\text { views }\end{array}$ & 2.14 & $20: 24: 00$ \\
\hline $\begin{array}{l}\text { Average } \\
\text { time on } \\
\text { site }\end{array}$ & 0:04:14 & $05: 02: 53$ \\
\hline New Visits & 270 & 17,420 \\
\hline
\end{tabular}

three months, the results showed that our sites were being listed in the first 10 pages in SERP. One of our sites started appearing on top of the first page and another appeared at the third ranking. Rest of the websites were facing a tough competition yet these were appearing on 4th and 6th page in the Google search. The reason for this could be that the traffic on those sites was less (just few hundred), especially the number of unique visitors and returning visitors were constantly reducing. After implementation our proposed framework, we noticed that all the statistics were showing upward trend. In table 2, we present statistical reports of one of our projects that we collected after three months.

The experimental results showed that on-page optimization is relatively better for improving page rank while off-page optimization is better for getting top position in SERP against some specific keywords. Similarly, we noticed that social media also plays a great role in traffic generation and branding.

Egria and Bayrak [3] implemented SEO on the site "indir.com" and reported that after four months of SEO activities they were able to achieve third site ranking in the Google search and their number of visitors increased to 35,000 . The average duration of each user's visit was estimated to be $2^{1 / 2} \mathrm{~min}$. Whereas, one of the sites optimized through our framework acquired first ranking and another achieved third ranking in the SERP just in three months duration. Tough the number of visitors on our site 
increased to 22,916 but the average duration of each user's visit was more than $5 \mathrm{~min}$. Thus, a comparison of the results with a similar study shows that our framework was more consistent and adhered to the user requirements more effectively. The additional key factors that influence SEO include speed of site opening, website session duration, bounce rate and usability. Our study also performed promisingly better for these additional parameters. Speed of site opening was increased by $40 \%$, average website session durations increased by two-fold and bounce rate decreased from $57 \%$ to $34 \%$, which indicate a significant improvement achieved through our SEO framework.

From managerial perspective, the proposed framework saves a lot of money as compared to the sponsored search advertising where retailers have to pay a fee to the search engines so that their sites get top ranking and are displayed alongside the organic web search results. Advertisers generally auction search keywords and charge click-through rates from the companies to maximize their profits. Our framework can thus save revenues of the companies as it serves as an alternate to investing on sponsored search, payper-click and search keywords bidding, etc. Also, the sites optimized through our framework would bring more revenues for the retailers as more and more customers would visit their sites due to its high visibility.

However, there are certain limitations of the proposed SEO framework. Firstly, the implementation of the proposed framework requires an extensive knowledge on content writing, therefore, SEO experts need to be trained on SEO approaches and should possess advanced analytical skills. Secondly, the keyword density needs a lot of deliberations to decide which set of keyword density would work. This make the proposed SEO process a time consuming job. Our approach necessitates that SEO experts should also demonstrate creative skills as sometimes a succinct and fascinating post can do wonders as compared to the effect that hundreds of SEO related words produced when inserted into the site for optimization purpose. The guidelines proposed in our framework are not exhaustive and need to be improved from time to time to tackle strategies of the competitors. Nonetheless, the last point is not at all a caveat of our framework because SEO is a continuous process rather than a project.

\section{Conclusion and future work}

In the current era, SEO is necessary for companies to increase their businesses. It is important to use new techniques for SEO to make a website popular and acquire higher rank in search results. SEO makes site highly visible if the keywords are found correctly by the crawler. In this paper, we have discussed the overall SEO process and proposed a meticulous framework for different stages of SEO. A better rank of website may increase the business and attract more visitors. We have also highlighted some key challenges of search engine optimization in this study. As a future dimension to this research, we aim to develop an efficient tool for keywords and backlinks suggestions to make these processes easier and time saving because most of the time the SEO project is spent on keywords research and identifying the relevant sites for backlinks generation.

\section{References}

[1] Shih B Y, Chen C Y and Chen Z S 2013 Retracted: an empirical study of an internet marketing strategy for search engine optimization. Human Factors and Ergonomics in Manufacturing and Service Industries 23(6): 528-540

[2] Andrews D, Nonnecke B and Preece J 2003 Electronic survey methodology: A case study in reaching hard-to-involve Internet users. International journal of Human-Computer Interaction 16(2): 185-210

[3] Egria G and Bayrak C 2014 The role of search engine optimization on keeping the user on the site. Procedia Computer Science 36: 335-342

[4] Yang Z, Shi Y and Wang B 2015 Search engine marketing, financing ability and firm performance in E-commerce. Procedia Computer Science 55: 1106-1112

[5] Yalçın N and Köse U 2010 What is search engine optimization: SEO? Procedia-Social and Behavioral Sciences 9: 487-493

[6] Malaga R A 2010 Search engine optimization—black and white hat approaches. Advances in Computers 78: 1-39

[7] Cui M and Hu S 2011 Search engine optimization research for website promotion. In: Information Technology, Computer Engineering and Management Sciences (ICM), 2011 International Conference on (Vol. 4), pp. 100-103. IEEE

[8] Wang F, Li Y and Zhang Y 2011 An empirical study on the search engine optimization technique and its outcomes. In: 2nd International Conference on Artificial Intelligence, Management Science and Electronic Commerce (AIMSEC), 2011, pp. 2767-2770. IEEE

[9] Kumar S M, Revathy P and Vijayalakshmi K 2009 Implementation of two-tier link extractor in optimized search engine filtering system. In: IEEE International Conference on Internet Multimedia Services Architecture and Applications (IMSAA), 2009, pp. 1-4. IEEE

[10] Li-Hsing H, Jui-Chen H, Meng-Huang L and Hui-Yi H 2010 The application of search engine optimization for internet marketing: an example of the motel websites. In: The 2nd International Conference on Computer and Automation Engineering (ICCAE), 2010 (Vol. 1), pp. 380-383. IEEE

[11] Chung P T, Chung S H and Hui C K 2012 A web server design using search engine optimization techniques for web intelligence for small organizations. In: Systems, Applications and Technology Conference (LISAT), 2012 IEEE Long Island, pp. 1-6. IEEE

[12] Yunfeng M 2010 A study on tactics for corporate website development aiming at search engine optimization. In: Second International Workshop on Education Technology and Computer Science (ETCS), 2010 (Vol. 3), pp. 673-675. IEEE 
[13] Elgharabawy M A and Ayu M A 2011 Web content accessibility and its relation to Webometrics ranking and search engines optimization. In: International Conference on Research and Innovation in Information Systems (ICRIIS), 2011, pp. 1-6. IEEE

[14] Zhu C and Wu G 2011 Research and analysis of search engine optimization factors based on reverse engineering. In: Third International Conference on Multimedia Information Networking and Security (MINES), 2011, pp. 225-228. IEEE

[15] Rajaram M and Vadivu S 2010 Web caching in Semantic Web based multiple search engines. In: IEEE International Conference on Computational Intelligence and Computing Research (ICCIC), 2010, pp. 1-7. IEEE

[16] Wang S, Xu K, Zhang Y and Li F 2011 Search engine optimization based on algorithm of BP neural networks. In: Seventh International Conference on Computational Intelligence and Security (CIS), 2011, pp. 390-394. IEEE

[17] Yuan S, Mu B and Zhang X 2010 Implementation for fulltext search and rank optimization in e-commerce. In: International Conference on Computer Design and Applications (ICCDA), 2010 (Vol. 2), pp. 2-196. IEEE

[18] Somani A and Suman U 2011 Counter measures against evolving search engine spamming techniques. In: $3 r d$ International Conference on Electronics Computer Technology (ICECT), 2011 (Vol. 6), pp. 214-217. IEEE

[19] Egele M, Kolbitsch C and Platzer C 2011 Removing web spam links from search engine results. Journal in Computer Virology 7(1): 51-62
[20] Ayanso A and Karimi A 2015 The moderating effects of keyword competition on the determinants of ad position in sponsored search advertising. Decision Support Systems 70: 42-59

[21] Ortiz-Cordova A, Yang Y and Jansen B J 2015 External to internal search: Associating searching on search engines with searching on sites. Information Processing and Management 51(5): 718-736

[22] Amin G R and Emrouznejad A 2011 Optimizing search engines results using linear programming. Expert Systems with Applications 38(9): 11534-11537.

[23] Mavridis T and Symeonidis A L 2015 Identifying valid search engine ranking factors in a Web 2.0 and Web 3.0 context for building efficient SEO mechanisms. Engineering Applications of Artificial Intelligence 41: 75-91

[24] Pan B 2015 The power of search engine ranking for tourist destinations. Tourism Management 47: 79-87

[25] Berman R and Katona Z 2013 The role of search engine optimization in search marketing. Marketing Science 32(4): 644-651

[26] Höchstötter N and Lewandowski D 2009 What users seeStructures in search engine results pages. Information Sciences 179(12): 1796-1812

[27] Beel J, Gipp B and Wilde E 2009 Academic Search Engine Optimization (ASEO) Optimizing Scholarly Literature for Google Scholar \& Co. Journal of scholarly publishing 41(2): 176-190

[28] Giomelakis D and Veglis A 2015 Employing search engine optimization techniques in online news articles. Studies in Media and Communication 3(1): 22-33 